\title{
Qualitative properties of mathematical model of English language education
}

\author{
Atena Ghasemabadi ${ }^{* *}$ and Nahid Soltanian²
}

"Correspondence:

ghasemabadi.math@gmail.com

${ }^{1}$ Esfarayen University of Technology, Esfarayen, North Khorasan, Iran

Full list of author information is

available at the end of the article

\section{Springer}

\begin{abstract}
This paper presents a mathematical model that examines the impacts of traditional and modern educational programs. We calculate two reproduction numbers. By using the Chavez and Song theorem, we show that backward bifurcation occurs. In addition, we investigate the existence and local and global stability of boundary equilibria and coexistence equilibrium point and the global stability of the coexistence equilibrium point using compound matrices.
\end{abstract}

MSC: $37 \mathrm{C} 75 ; 37 \mathrm{~N} 25 ; 39 \mathrm{~A} 11 ; 65 \mathrm{C} 20 ; 65 \mathrm{~L} 12$

Keywords: Mathematical modeling; Global stability; Backward bifurcation; Foreign language education

\section{Introduction}

Learning a foreign language has always been one main issue for individuals who are living in a globalized world. To achieve this aim, the individuals have spent expense, energy, and time.

The main problem in foreign language education relates to how to teach it: traditional or modern education. The classes are still traditional i.e., teacher-centered, and the methods used for teaching do not attend to the individual's personal needs. Moreover, these methods do not truly support the ambition to develop team working abilities among the students. Not surprisingly, a redesign of the situation is necessary to improve it. Modern education (computer-aided procedures) can function as a supplement to traditional education. Thanks to advancements in educational technology such as computers and social media, learning a language does not seem the same painstaking task as before, since as time passes it has turned out to be quite enhanced moving from teacher-centered to learner-centered methods.

Firstly, some do not succumb to the reality of the benefits they can have from emerging technology, and secondly, there are others who fascinatingly hurry to use technology with no learning discipline. Modern education is not devoid of problems. The major problem refers to the point that normally sufficient computers are not available. The learners may also be short of time to use computers for learning. Moreover, in this system of education, the learner requires some skills in the appropriate use of computers.

(c) The Author(s) 2021. This article is licensed under a Creative Commons Attribution 4.0 International License, which permits use, sharing, adaptation, distribution and reproduction in any medium or format, as long as you give appropriate credit to the original author(s) and the source, provide a link to the Creative Commons licence, and indicate if changes were made. The images or other third party material in this article are included in the article's Creative Commons licence, unless indicated otherwise in a credit line to the material. If material is not included in the article's Creative Commons licence and your intended use is not permitted by statutory regulation or exceeds the permitted use, you will need to obtain permission directly from the copyright holder. To view a copy of this licence, visit http://creativecommons.org/licenses/by/4.0/. 
The majority of educational programs can be applied in certain conditions. For example, the software which works in the Windows environment cannot be used in the IOS environment. The opposite case is also true.

Another problem with modern education is that the software and hardware applications and systems are usually expensive and having access to them is difficult. Some learners do not easily accept technology and prefer to be taught through traditional methods.

Levine [5] and other scholars state that although in both methods of education, i.e. traditional and modern, the learners can enjoy the same level of language learning, this level can be achieved in less time in modern education.

We can consider individuals who tend to learn a foreign language either through traditional methods or modern methods according to their abilities and interests. In addition, they can switch between traditional and modern education. Individuals who fail to learn English as a foreign language (EFL) can participate in training again. The present study aims to compare the two educational systems of traditional and modern by using mathematical modeling to examine which system is more effective. This modeling becomes the development of epidemic model with two strains and superinfection $[7,8]$. We use the mathematical modeling of Lotka-Volterra model see [3, 4].

At first in Sect. 2, the researchers present the model and define two basic reproduction numbers. They examine some preliminaries such as boundedness and the positivity of the solutions of the model. In Sect. 3, the existence of equilibria and local and global asymptotical stable is investigated. In Sect. 4, the occurrence of backward bifurcation is shown by using the theorem of Castillo-Chavez and Song [2]. In Sect. 5, they use a geometric approach, introduced by Muldowney and $\mathrm{Li}$ [6], and show that the nontrivial positive equilibrium is globally stable. Finally, a numerical simulation is carried out to support the analytical results.

\section{Mathematical model}

In this paper, the model of learning the English language by university students has been considered. It has the following compartments: $S(t)$ is the number of susceptible individuals who tend to participate in language learning classes, $C(t)$ is the number of students who have selected modern education (computer-aided procedures). $T(t)$ is the number of students who have selected traditional education. $F(t)$ is the number of students who have successfully completed the course.

Class $T$ selected traditional education due to several reasons including not having easy access to computer within the class, not being skilled enough in working with computer, the high expense of educational software, and the teachers' unwillingness or their less proficiency to work with computer.

Class $C$ selected modern education due to the problems of traditional education. With the passage of time, an individual who has been present in the traditional class of $T$ may prefer to learn English through modern education maybe because he/she has learned the necessary skills for working with a computer, and is now able to overcome the problems of modern education. Hence, he/she may be transferred to class C with the rate of $\delta_{1}$. Another case may happen too. An individual in the modern class may come to the conclusion that the traditional class is more suitable for him/her. So, he/she is transferred to class $\mathrm{T}$ with the rate of $\delta_{2}$. In mathematical modeling, the rate of death is considered as $\mu$. It is hypothesized that in modern education the individuals with the rate of $\alpha_{1}$ and in 
Figure 1 Model diagram

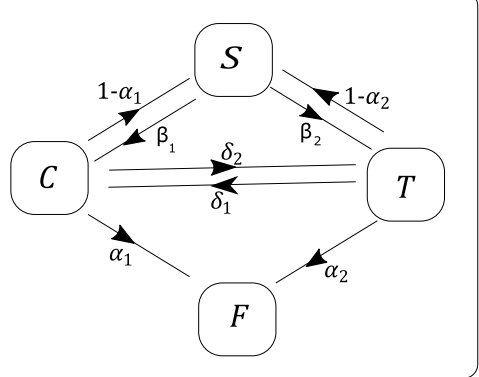

the traditional education the individuals with the rate of $\alpha_{2}$ are successful in learning English. With the rates of $1-\alpha_{1}$ and $1-\alpha_{2}$, the individuals who have not been successful in learning English are transferred to the class of susceptible individuals. We also assume that susceptible individuals enter the classes of modern and traditional education at the rates of $\frac{\beta_{1} C}{N}$ and $\frac{\beta_{2} T}{N}$, respectively.

Based on the above assumptions and model diagram in the Fig. 1, the proposed model is formulated as the following model:

$$
\left\{\begin{array}{l}
\dot{S}=\Lambda-\beta_{1} \frac{S C}{N}-\beta_{2} \frac{S T}{N}-\mu S+\left(1-\alpha_{1}\right) C+\left(1-\alpha_{2}\right) T, \\
\dot{C}=\beta_{1} \frac{S C}{N}-\delta_{2} \beta_{2} \frac{T C}{N}+\delta_{1} \beta_{1} \frac{T C}{N}-\left(\mu+\alpha_{1}\right) C-\left(1-\alpha_{1}\right) C, \\
\dot{T}=\beta_{2} \frac{S T}{N}+\delta_{2} \beta_{2} \frac{T C}{N}-\delta_{1} \beta_{1} \frac{T C}{N}-\left(\mu+\alpha_{2}\right) T-\left(1-\alpha_{2}\right) T, \\
\dot{F}=\alpha_{1} C+\alpha_{2} T-\mu F,
\end{array}\right.
$$

The parameters in the model (2.1) are as follows:

- $N$ : Size of the total population.

- $S$ : The number of susceptible individuals who tend to participate in English language class.

- $T$ : The number of individuals who tend to learn English language through traditional methods not computer-aided procedures.

- $C$ : The number of individuals who tend to learn English language via computer-aided procedures (modern education).

- F: Individuals who have finished the course successfully.

- $\Lambda$ : The rate at which recruits enter the susceptible population.

- $\beta_{1}$ : The probability that a susceptible individual uses traditional education to learn English language.

- $\beta_{2}$ : The probability that a susceptible individual uses modern education (computer-aided procedures) to learn English language.

- $\mu$ : The natural death rate of the general population.

- $\delta_{1}$ : The rate at which a language learner who uses traditional education turns to a language learner who uses modern education (computer-aided procedures).

- $\delta_{2}$ : The rate at which a language learner who uses modern education (computer-aided procedures) turns to a language learner who uses traditional education.

- $\alpha_{1}$ : The rate of ending the traditional course by a language learner successfully.

- $\alpha_{2}$ : The rate of ending the modern course by a language learner successfully.

The total population $N(t)=S(t)+C(t)+T(t)+F(t)$ satisfies the following relation:

$$
\frac{d N}{d t}=\Lambda-\mu N
$$


hence $\lim \sup _{t \rightarrow \infty} N(t)=\frac{\Lambda}{\mu}$. Therefore the set

$$
\Omega=\left\{(S, C, T, F): S>0, C>0, T>0, F>0, S+C+T+F \leq \frac{\Lambda}{\mu}\right\}
$$

is a positively invariant set for (2.1). Therefore the dynamics of the model can be studied only in $\Omega$.

We define two reproduction numbers:

$$
R_{1}=\frac{\beta_{1}}{\mu+1}, \quad R_{2}=\frac{\beta_{2}}{\mu+1}, \quad R_{0}=\max \left\{R_{1}, R_{2}\right\}
$$

Let

$$
s=\frac{S}{N}, \quad c=\frac{C}{N}, \quad g=\frac{T}{N}, \quad f=\frac{F}{N},
$$

where $s=1-c-g-f$. Hence system (2.1) is converted to the following system:

$$
\left\{\begin{array}{l}
\dot{c}=\beta_{1}\left(c-c^{2}-c g-c f\right)+\left(\delta_{1} \beta_{1}-\delta_{2} \beta_{2}\right) c g-(\mu+1) c, \\
\dot{g}=\beta_{2}\left(g-g^{2}-c g-f g\right)+\left(\delta_{2} \beta_{2}-\delta_{1} \beta_{1}\right) c g-(\mu+1) g, \\
\dot{f}=\alpha_{1} c+\alpha_{2} g-\mu f .
\end{array}\right.
$$

Now, we prove the positivity of the solutions.

Proposition 2.1 If the initial conditions are positive, i.e., $c(0)>0, g(0)>0$ and $f(0)>0$, then the solution $(c(t), g(t), f(t))$ of the system is positive for all $t \geq 0$.

Proof Let $(c(t), g(t), f(t))$ be a solution of the system with $c(0)>0, g(0)>0$ and $f(0)>0$. Assume the conclusion is not true, then there is a $t^{*}>0$ such that

$$
\min \left\{c\left(t^{*}\right), g\left(t^{*}\right), f\left(t^{*}\right)\right\}=0
$$

and

$$
\min \{c(t), g(t), f(t)\}>0, \quad \forall t \in\left[0, t^{*}\right)
$$

If $\min \left\{c\left(t^{*}\right), g\left(t^{*}\right), f\left(t^{*}\right)\right\}=f\left(t^{*}\right)$, then we have

$$
\dot{f} \geq-\mu f(t), \quad \forall t \in\left[0, t^{*}\right)
$$

Therefore $0=f\left(t^{*}\right) \geq f(0) \exp (-\mu t)>0$, hence it is a contradiction. Similarly, if $\min \left\{c\left(t^{*}\right)\right.$, $\left.g\left(t^{*}\right), f\left(t^{*}\right)\right\}$ is equal to $c\left(t^{*}\right)$ or $g\left(t^{*}\right)$, then this leads to a contradiction.

\section{Existence and stability of equilibria $E_{0}, E_{1}$ and $E_{2}$}

System (2.5) has the following equilibria:

$$
E_{0}=(0,0,0)
$$




$$
\begin{aligned}
& E_{1}=(\widehat{c}, 0, \widehat{f})=\left(\frac{\mu}{\mu+\alpha_{1}}\left(1-\frac{1}{R_{1}}\right), 0, \frac{\alpha_{1}}{\mu+\alpha_{1}}\left(1-\frac{1}{R_{1}}\right)\right), \\
& E_{2}=(0, \bar{g}, \bar{f})=\left(0, \frac{\mu}{\mu+\alpha_{2}}\left(1-\frac{1}{R_{2}}\right), \frac{\alpha_{2}}{\mu+\alpha_{2}}\left(1-\frac{1}{R_{2}}\right)\right) .
\end{aligned}
$$

The equilibria $E_{1}$ and $E_{2}$ exist provided that $R_{0}>1$.

Theorem 3.1 The education-free equilibrium $E_{0}$ is asymptotically stable provided that $R_{0}<1$.

Proof We need to be careful in computing the Jacobian. In particular, we need to keep in mind that $N(t)=S(t)+C(t)+T(t)+F(t)$. The Jacobian matrix of the system has the following form:

$$
J=\left[\begin{array}{lll}
a_{11} & a_{12} & a_{13} \\
a_{21} & a_{22} & a_{23} \\
a_{31} & a_{32} & a_{33}
\end{array}\right],
$$

where

$$
\begin{aligned}
& a_{11}=\beta_{1}\left(1-2 c+c^{2}-g+c g-f+f c\right)+\left(\delta_{1} \beta_{1}-\delta_{2} \beta_{2}\right)(g-c g \mu+1), \\
& a_{12}=\beta_{1}\left(-c+c g+c^{2}+c f\right)+\left(\delta_{1} \beta_{1}-\delta_{2} \beta_{2}\right)(c-c g), \\
& a_{13}=\beta_{1}\left(c^{2}+c g-c+c f\right)+\left(\delta_{2} \beta_{2}-\delta_{1} \beta_{1}\right) c g, \\
& a_{21}=\beta_{2}\left(-g+g^{2}+c g+g f\right)+\left(\delta_{2} \beta_{2}-\delta_{1} \beta_{1}\right)(g-c g), \\
& a_{22}=\beta_{2}\left(1-c+c g-2 g+g^{2}-f+f g\right)+\left(\delta_{2} \beta_{2}-\delta_{1} \beta_{1}\right)(c-c g)-(\mu+1), \\
& a_{23}=\beta_{2}\left(c g+g^{2}-g+g f\right)+\left(\delta_{1} \beta_{1}-\delta_{2} \beta_{2}\right) c g, \\
& a_{31}=\alpha_{1}, \\
& a_{32}=\alpha_{2}, \\
& a_{33}=-\mu .
\end{aligned}
$$

The Jacobian matrix at the equilibrium $E_{0}$ is

$$
J_{0}=J\left(E_{0}\right)=\left[\begin{array}{ccc}
\beta_{1}-(\mu+1) & 0 & 0 \\
0 & \beta_{2}-(\mu+1) & 0 \\
\alpha_{1} & \alpha_{2} & -\mu
\end{array}\right] .
$$

$J_{0}$ has the eigenvalues

$$
\begin{aligned}
& \lambda_{01}=\beta_{1}-(\mu+1)=\beta_{1}\left(1-\frac{1}{R_{1}}\right), \\
& \lambda_{02}=\beta_{2}-(\mu+1)=\beta_{2}\left(1-\frac{1}{R_{2}}\right), \\
& \lambda_{03}=-\mu .
\end{aligned}
$$

Since $R_{0}<1$, all eigenvalues are negative. Therefore $E_{0}$ is asymptotically stable. 
We use Lasalle's invariance principle and we show that the education-free $E_{0}$ is globally asymptotically stable.

Theorem 3.2 (Lasalle's invariance principle) Let $X^{*}$ be an equilibrium point for $X^{\prime}=F(X)$ and let $L: U \rightarrow \mathbb{R}$ be a Lyapunov function for $X^{*}$, where $U$ is an open set containing $X^{*}$. Let $P \subset U$ be a neighborhood of $X^{*}$ that is closed and bounded. Suppose that $P$ is positively invariant and that there is no entire solution in $P-X^{*}$ on which $L$ is constant. Then $X^{*}$ is asymptotically stable, and $P$ is contained in the basin of attraction of $X^{*}$.

Theorem 3.3 The education-free equilibrium $E_{0}$ is globally asymptotically stable provided that $R_{0}<1$.

Proof Consider the following Lyapunov function on $\mathbb{R}_{+}^{3}$ :

$$
V=c+g \text {. }
$$

It is easy to see that $V=0$ at the disease-free equilibrium. We compute the derivative of $V$ with respect to $t$ :

$$
\begin{aligned}
\frac{d V}{d t}= & c^{\prime}+g^{\prime} \\
= & \beta_{1}\left(c-c^{2}-c g-f c\right)+\left(\delta_{1} \beta_{1}-\delta_{2} \beta_{2}\right) c g-(\mu+1) c \\
& +\beta_{2}\left(g-g^{2}-c g-f g\right)+\left(\delta_{2} \beta_{2}-\delta_{1} \beta_{1}\right) c g-(\mu+1) g \\
= & \beta_{1} c\left(1-\frac{1}{R_{1}}\right)+\beta_{1}\left(-c^{2}-c g-f c\right) \\
& +\beta_{2} g\left(1-\frac{1}{R_{2}}\right)+\beta_{2}\left(-c g-g^{2}-f g\right) .
\end{aligned}
$$

Since $R_{0}<1$, then $d V$ is nonpositive. Hence the education-free equilibrium $E_{0}$ is globally asymptotically stable by the Lassalle invariance principle.

In the following theorem, we get the conditions that the equilibrium $E_{1}=(\hat{c}, 0, \hat{f})$ is locally asymptotically stable.

\section{Theorem 3.4 Let}

$$
\widehat{R_{2}^{1}}=\frac{\beta_{2}(1-\hat{c}-\hat{f})+\delta_{2} \beta_{2} \hat{c}}{\delta_{1} \beta_{1} \hat{c}+\mu+1} .
$$

The equilibrium $E_{1}=(\hat{c}, 0, \hat{f})$ is locally asymptotically stable if and only if $\widehat{R_{2}^{1}}<1$.

Proof The local stability of the equilibrium $E_{1}$ is given by the Jacobian matrix at $E_{1}$ :

$$
J_{1}=J\left(E_{1}\right)=\left[\begin{array}{ccc}
b_{11} & b_{12} & b_{13} \\
0 & b_{22} & 0 \\
\alpha_{1} & \alpha_{2} & -\mu
\end{array}\right] \text {, }
$$


where

$$
\begin{aligned}
& b_{11}=\beta_{1}\left(1-2 \widehat{c}+\widehat{c}^{2}-\widehat{f}+\widehat{f} \widehat{c}\right)-(\mu+1), \\
& b_{12}=\beta_{1}\left(-\widehat{c}+\widehat{c}^{2}+\widehat{f} \widehat{c}\right)+\left(\delta_{1} \beta_{1}-\delta_{2} \beta_{2}\right) \widehat{c}, \\
& b_{13}=\beta_{1}\left(\widehat{c}^{2}-\widehat{c}+\widehat{c} \widehat{f}\right) \\
& b_{22}=\beta_{2}(1-\widehat{c}-\widehat{f})+\left(\delta_{2} \beta_{2}-\delta_{1} \beta_{1}\right) \widehat{c}-(\mu+1) .
\end{aligned}
$$

An eigenvalue of matrix $J_{1}$ is

$$
\lambda_{11}=\beta_{2}(1-\widehat{c}-\widehat{f})+\left(\delta_{2} \beta_{2}-\delta_{1} \beta_{1}\right) \widehat{c}-(\mu+1) .
$$

The other eigenvalues are the eigenvalues of the following submatrix:

$$
J_{11}=\left[\begin{array}{cc}
\beta_{1}\left(1-2 \widehat{c}+\widehat{c}^{2}-\widehat{f}+\widehat{f} \widehat{c}\right)-(\mu+1) & \beta_{1}\left(\widehat{c}^{2}-\widehat{c}+\widehat{c} \widehat{f}\right) \\
\alpha_{1} & -\mu
\end{array}\right] .
$$

Matrix $J_{11}$ has eigenvalues with the negative real parts if $\operatorname{Tr}\left(J_{11}\right)<0$ and $\operatorname{det}\left(J_{11}\right)>0$. We can easily see that

$$
\operatorname{Tr}\left(J_{11}\right)=\beta_{1}\left(1-2 \widehat{c}+\widehat{c}^{2}-\widehat{f}+\widehat{f} \widehat{c}\right)-2 \mu-1
$$

By replacing

$$
\widehat{c}=\frac{\mu}{\mu+\alpha_{1}}\left(1-\frac{1}{R_{1}}\right), \quad \widehat{f}=\frac{\alpha_{1}}{\mu+\alpha_{1}}\left(1-\frac{1}{R_{1}}\right),
$$

in Eq. (3.2), since $R_{1}>1$, we get

$$
\begin{aligned}
& \operatorname{Tr}\left(J_{11}\right)=\frac{1}{\beta_{1}\left(\mu+\alpha_{1}\right)}\left(\mu(\mu+1)\left(\frac{1}{R_{1}}-1\right)-\beta_{1} \mu^{2}-\alpha_{1} \beta_{1} \mu\right)<0, \\
& \operatorname{det}\left(J_{11}\right)=\mu(\mu+1)\left[1-\frac{1}{R_{1}}\right]>0 .
\end{aligned}
$$

Hence, stability $E_{1}$ is determined by the sign of the eigenvalue $\lambda_{11}$,

$$
\lambda_{11}=\beta_{2}(1-\widehat{c}-\widehat{f})+\left(\delta_{2} \beta_{2}-\delta_{1} \beta_{1}\right) \widehat{c}-(\mu+1)<0 .
$$

The equilibrium $E_{1}$ will be locally asymptotically stable if and only if $\lambda_{11}<0$, that is,

$$
\widehat{R_{2}^{1}}=\frac{\beta_{2}(1-\hat{c}-\hat{f})+\delta_{2} \beta_{2} \hat{c}}{\delta_{1} \beta_{1} \hat{c}+\mu+1}<1 .
$$

Therefore, the equilibrium $E_{1}=(\hat{c}, 0, \hat{f})$ is locally asymptotically stable.

Theorem 3.5 Suppose $2 \alpha_{1}=2 \alpha_{1}=\beta_{1}=\beta_{2}$. The equilibrium $E_{1}=(\hat{c}, 0, \hat{f})$ is globally asymptotically stable provided that $\widehat{R_{2}^{1}}<1$. 
Proof We consider the following Lyapunov function on $\mathbb{R}_{+}^{3}$ :

$$
V=K_{1}\left(c-\widehat{c}-\widehat{c} \operatorname{Ln}\left(\frac{c}{\widehat{c}}\right)\right)+K_{2} g+K_{3}(f-\widehat{f})^{2} .
$$

It is not hard to see that $V=0$ at $E_{1}$. We take the derivative of $V$ with respect to $t$ :

$$
\begin{aligned}
\frac{d V}{d t}= & K_{1}(c-\widehat{c}) \frac{c^{\prime}}{c}+K_{2} g^{\prime}+2 K_{3}(f-\widehat{f}) f^{\prime} \\
= & K_{1}(c-\widehat{c})\left(\beta_{1}(1-c-g-f)+\left(\delta_{1} \beta_{1}-\delta_{2} \beta_{2}\right) g-(\mu+1)\right) \\
& +K_{2} g\left(\beta_{2}(1-c-g-f)+\left(\delta_{2} \beta_{2}-\delta_{1} \beta_{1}\right) c-(\mu+1)\right) \\
& +2 K_{3}(f-\widehat{f})\left(\alpha_{1} c-\alpha_{2} g-\mu f\right) \\
= & -K_{1} \beta_{1}(c-\widehat{c})^{2}-K_{2} \beta_{2} g^{2}-2 K_{3} \mu(f-\widehat{f})^{2} \\
& +K_{2} g\left(\widehat{R_{2}^{1}}-1\right)+(f-\hat{f}) g\left(-K_{2} \beta_{2}+2 K_{3} \alpha_{2}\right) \\
& +(c-\hat{c}) g\left(-K_{2} \beta_{2}-K_{1} \beta_{1}+K_{1} \delta-K_{2} \delta\right) \\
& +(c-\widehat{c})(f-\hat{f})\left(-K_{1} \beta_{1}+2 K_{3} \alpha_{1}\right) .
\end{aligned}
$$

We evaluate the coefficients $K_{1}, K_{2}, K_{3}$, such that the coefficients of $g(f-\hat{f})$ and $(c-\hat{c})(f-\hat{f})$ are equal to zero:

$$
\begin{aligned}
& K_{1}=K_{2}, \\
& K_{3}=\frac{\beta_{1}}{2 \alpha_{1}} K_{1}=\frac{\beta_{2}}{2 \alpha_{2}} K_{2} .
\end{aligned}
$$

The following inequality has been obtained from the equation for $d V / d t$ and by using the inequalities $a^{2}+b^{2} \geq 2 a b$ and $a^{2}+b^{2} \geq-2 a b$ :

$$
\frac{d V}{d t} \leq-2 K_{3} \mu(f-\widehat{f})^{2}+K_{2} g\left(\widehat{R}_{2}^{1}-1\right) .
$$

If $\widehat{R_{2}^{1}}<1$, then $\frac{d V}{d t}$ is negative. Therefore, by the Lasalle invariance principle, the equilibrium $E_{1}$ is globally asymptotically stable.

The proof of locally and globally asymptotically stability of $E_{2}$ is similar to the one discussed above. For this purpose, we define

$$
\widehat{R_{1}^{2}}=\frac{\beta_{1}(1-\bar{g}-\bar{f})+\delta_{1} \beta_{1} \bar{g}}{\delta_{1} \beta_{1} \bar{g}+\mu+1}
$$

Now, we get an equilibrium in which both types of educational methods are present. This equilibrium is a nontrivial solution of the following system:

$$
\left\{\begin{array}{l}
\beta_{1}(1-c-g-f)+\left(\delta_{1} \beta_{1}-\delta_{2} \beta_{2}\right) g=\mu+1 \\
\beta_{2}(1-g-c-f)+\left(\delta_{2} \beta_{2}-\delta_{1} \beta_{1}\right) c=\mu+1, \\
\alpha_{1} c+\alpha_{2} g-\mu f=0 .
\end{array}\right.
$$


System (3.5) gives the following values:

$$
\begin{aligned}
c^{*} & =\frac{\beta_{2}\left(\mu+\alpha_{2}\right)(\mu+1)\left(\widehat{R_{1}^{2}}-1\right)}{\delta\left(\left(\beta_{2}-\beta_{1}\right) \mu+\left(\alpha_{1} \beta_{2}-\alpha_{2} \beta_{1}\right)+\delta \mu\right)}, \\
g^{*} & =\frac{\beta_{1}\left(\mu+\alpha_{1}\right)(\mu+1)\left(\widehat{R_{2}^{1}}-1\right)}{\delta\left(\left(\beta_{2}-\beta_{1}\right) \mu+\left(\alpha_{1} \beta_{2}-\alpha_{2} \beta_{1}\right)+\delta \mu\right)}, \\
f^{*} & =\frac{\left(\mu+\alpha_{2}\right)(\mu+1) \alpha_{1}\left(\widehat{R_{1}^{2}}-1\right)+\alpha_{2}\left(\widehat{R_{2}^{1}}-1\right)\left(\mu+\alpha_{1}\right)(\mu+1)}{\delta\left(\left(\beta_{2}-\beta_{1}\right) \mu+\left(\alpha_{1} \beta_{2}-\alpha_{2} \beta_{1}\right)+\delta \mu\right)},
\end{aligned}
$$

where $\delta=\delta_{1} \beta_{1}-\delta_{2} \beta_{2}$. Therefore the following theorem holds.

Theorem 3.6 Suppose $\widehat{R_{2}^{1}}<1$ and $\widehat{R_{1}^{2}}<1$. The equilibrium $E_{3}=\left(c^{*}, t^{*}, f^{*}\right)$ exists provided that one of the following conditions holds:

1) $\delta>0, \beta_{1}<\beta_{2}$ and $\alpha_{2}<\alpha_{1}$,

2) $\delta<0, \beta_{2}<\beta_{1}$ and $\alpha_{1}<\alpha_{2}$.

\section{Backward bifurcation}

Initially, we will state Castillo-Chavez and Song theorem, then we show that backward bifurcation occurs.

Theorem 4.1 (Castillo-Chavez and Song theorem) Assume

A1: $A=D_{x} f(0,0)=\left(\frac{\partial f_{i}}{\partial x_{j}}(0,0)\right)$ is the linearization matrix of the following system around the equilibrium 0 with $\phi$ evaluated at 0 (zero is a simple eigenvalue of $A$ and all other eigenvalues of $A$ have negative real parts):

$$
\frac{d x}{d t}=f(x, \phi), \quad f: \mathbb{R}^{n} \times \mathbb{R} \rightarrow \mathbb{R} \text { and } f \in \mathbb{C}^{2}\left(\mathbb{R}^{n} \times \mathbb{R}\right)
$$

A2: Matrix A has a nonnegative right eigenvector $w$ and a left eigenvector $v$ corresponding to the zero eigenvalue.

Let $f_{k}$ be the kth component of $f$ and

$$
\begin{aligned}
& \boldsymbol{a}=\sum_{k, i, j=1}^{3} v_{k} w_{i} w_{j} \frac{\partial^{2} F_{k}}{\partial x_{i} \partial x_{j}}(0,0), \\
& \boldsymbol{b}=\sum_{k, i=1}^{3} v_{k} w_{i} \frac{\partial^{2} F_{k}}{\partial x_{i} \partial \varphi}(0,0) .
\end{aligned}
$$

The local dynamics of (4.1) around 0 are totally determined by $a$ and $b$.

i. $a>0, b>0$. When $\phi<0$ with $|\phi| \ll 1,0$ is locally asymptotically stable, and there exists a positive unstable equilibrium; when $0<\phi \ll 1,0$ is unstable and there exists a negative and a locally asymptotically stable equilibrium.

ii. $a<0, b>0$. When $\phi<0$ with $|\phi| \ll 1,0$ is unstable; when $0<\phi \ll 1,0$ is locally asymptotically stable, and there exists a positive unstable equilibrium.

iii. $a>0, b<0$. When $\phi<0$ with $|\phi| \ll 1,0$ is unstable, and there exists a locally asymptotically stable negative equilibrium; when $0<\phi \ll 1,0$ is stable, and a positive unstable equilibrium appears. 
iv. $a<0, b>0$. When $\phi$ changes from negative to positive, 0 changes its stability from stable to unstable. Correspondingly a negative unstable equilibrium becomes positive and locally asymptotically stable.

We apply the theorem of Castillo-Chavez and Song to show that in system backward bifurcation occurs in the following cases:

Case 1. $R_{1}=1$ and $R_{2}<1$.

Since $R_{1}=1$, we can compute $\beta_{1}$ as

$$
\beta_{1}=\beta_{1}^{*}=\mu+1
$$

The eigenvalues of $J\left(E_{0}, \beta_{1}^{*}\right)$ are $0,-\mu, \beta_{2}\left(1-\frac{1}{R_{2}}\right)$. Therefore, zero is simple and other eigenvalues are negative real numbers. If $R_{1}=1$, then $\lambda_{1}=0$ and corresponding right and left eigenvalues are $w=\left(1,0, \frac{\alpha_{1}}{\mu}\right)$ and $v=(1,0,0)$. We rewrite the system $(2.5)$ as follows:

$$
\left\{\begin{array}{l}
F_{1}\left(x_{1}, x_{2}, x_{3}\right)=\beta_{1}\left(x_{1}-x_{1}^{2}-x_{1} x_{2}-x_{1} x_{3}\right)+\left(\delta_{1} \beta_{1}-\delta_{2} \beta_{2}\right) x_{1} x_{2}-(\mu+1) x_{1} \\
F_{2}\left(x_{1}, x_{2}, x_{3}\right)=\beta_{2}\left(x_{2}-x_{2}^{2}-x_{1} x_{2}-x_{3} x_{2}\right)+\left(\delta_{2} \beta_{2}-\delta_{1} \beta_{1}\right) x_{1} x_{2}-(\mu+1) x_{2} \\
F_{3}\left(x_{1}, x_{2}, x_{3}\right)=\alpha_{1} x_{1}+\alpha_{2} x_{2}-\mu x_{3} .
\end{array}\right.
$$

Now, we compute the quantities $a$ and $b$ in the following form:

$$
\begin{aligned}
\mathbf{a} & =\sum_{k, i, j=1}^{3} v_{k} w_{i} w_{j} \frac{\partial^{2} F_{k}}{\partial x_{i} \partial x_{j}} \\
& =\sum_{i, j=1}^{3} w_{i} w_{j} \frac{\partial^{2} F_{1}}{\partial x_{i} \partial x_{j}}=-2 \beta_{1}^{*}+\left(\frac{\alpha_{1}}{\mu}\right)\left(-2 \beta_{1}^{*}\right)=\frac{-2 \beta_{1}^{*}\left(\alpha_{1}+\mu\right)}{\mu}<0 \\
\mathbf{b} & =\sum_{k, i=1}^{3} v_{k} w_{i} \frac{\partial^{2} F_{k}}{\partial x_{i} \partial \varphi}\left(E_{0}, \beta_{1}^{*}\right)=\sum_{i=1}^{3} w_{i} \frac{\partial^{2} F_{1}}{\partial x_{i} \partial \varphi}(0,0)=1
\end{aligned}
$$

Case 2. $R_{2}=1$ and $R_{1}<1$.

From $R_{2}=1$, we conclude that

$$
\beta_{2}=\beta_{2}^{*}=\mu+1 \text {. }
$$

The Jacobian matrix $J\left(E_{0}, \beta_{2}^{*}\right)$ has eigenvalues $0,-\mu, \beta_{1}\left(1-\frac{1}{R_{1}}\right)$. Therefore zero is simple, since $R_{1}<1$ other eigenvalues are negative real numbers. The right and left eigenvectors of $J\left(E_{0}, \beta_{2}^{*}\right)$ associated with $\lambda_{1}=0$ are $w=\left(0,1, \frac{\alpha_{2}}{\mu}\right)$ and $v=(0,1,0)$, respectively. Hence

$$
\begin{aligned}
\mathbf{a} & =\sum_{k, i, j=1}^{3} v_{k} w_{i} w_{j} \frac{\partial^{2} F_{k}}{\partial x_{i} \partial x_{j}} \\
& =\sum_{i, j=1}^{3} w_{i} w_{j} \frac{\partial^{2} F_{1}}{\partial x_{i} \partial x_{j}}=-2 \beta_{2}^{*}+\left(\frac{\alpha_{2}}{\mu}\right)\left(-2 \beta_{2}^{*}\right)=\frac{-2 \beta_{2}^{*}\left(\alpha_{2}+\mu\right)}{\mu}<0, \\
\mathbf{b} & =\sum_{k, i=1}^{3} v_{k} w_{i} \frac{\partial^{2} F_{k}}{\partial x_{i} \partial \varphi}\left(E_{0}, \beta_{1}^{*}\right)=\sum_{i=1}^{3} w_{i} \frac{\partial^{2} F_{1}}{\partial x_{i} \partial \varphi}(0,0)=1 .
\end{aligned}
$$

Since $\mathbf{a}<0$ and $\mathbf{b}>0$, model (2.1) undergoes a backward bifurcation. 


\section{Global stability of the nontrivial positive equilibrium}

The second additive compound of the Jacobian matrix $J$ is the $3 \times 3$ matrix given by $J^{[2]}$ :

$$
M=J^{[2]}=\left[\begin{array}{lll}
M_{11} & M_{12} & M_{13} \\
M_{21} & M_{22} & M_{23} \\
M_{31} & M_{32} & M_{33}
\end{array}\right]=\left[\begin{array}{ccc}
a_{11}+a_{22} & a_{23} & -a_{13} \\
a_{32} & a_{11}+a_{33} & a_{12} \\
-a_{31} & a_{21} & a_{22}+a_{33}
\end{array}\right] \text {, }
$$

where

$$
\begin{aligned}
M_{11}= & \beta_{1}\left(1-2 c+c^{2}-g+c g-f+f c\right)+\left(\delta_{1} \beta_{1}-\delta_{2} \beta_{2}\right) g \\
& -2(\mu+1)+\beta_{2}\left(1-c+c g-2 g+g^{2}-f+f g\right)+\left(\delta_{2} \beta_{2}-\delta_{1} \beta_{1}\right) c, \\
M_{12}= & \beta_{2}\left(c g+g^{2}-g+f g\right)+\left(\delta_{1} \beta_{1}-\delta_{2} \beta_{2}\right) c g, \\
M_{13}= & -\beta_{1}\left(c^{2}+c g-c+c f\right)+\left(\delta_{1} \beta_{1}-\delta_{2} \beta_{2}\right) c g, \\
M_{21}= & \alpha_{2}, \\
M_{22}= & \beta_{1}\left(1-2 c+c^{2}-g+c g-f+f c\right)+\left(\delta_{1} \beta_{1}-\delta_{2} \beta_{2}\right)(g-c g)-2 \mu-1, \\
M_{23}= & \beta_{1}\left(-c+c g+c^{2}+c f\right)+\left(\delta_{1} \beta_{1}-\delta_{2} \beta_{2}\right)(c-c g), \\
M_{31}= & -\alpha_{1}, \\
M_{32}= & \beta_{2}\left(-g+g^{2}+c g+g f\right)+\left(\delta_{2} \beta_{2}-\delta_{1} \beta_{1}\right)(g-c g), \\
M_{33}= & \beta_{2}\left(1-c+c g-2 g+g^{2}-f+f g\right)+\left(\delta_{2} \beta_{2}-\delta_{1} \beta_{1}\right)(c-c g)-2 \mu-1 .
\end{aligned}
$$

Now, we use the geometric method for the global stability problem and prove sufficient conditions for the global stability of the nontrivial positive equilibrium(see [6]). We denote unit ball of $\mathbb{R}^{2}$ and its closure and boundary by $\mathcal{B}, \overline{\mathcal{B}}$ and $\partial \mathcal{B}$, respectively. The collection of all Lipschitzian functions from $X$ to $Y$ is denoted by $\operatorname{Lip}(X \rightarrow Y)$. A function $\phi \in \operatorname{Lip}(\overline{\mathcal{B}} \rightarrow$ $\Omega$ ) is considered as a simply connected and rectifiable surface in $\Omega \subseteq \mathbb{R}^{n}$. A rectifiable and closed curve in $\Omega$ is a function $\phi \in \operatorname{Lip}(\partial \mathcal{B} \rightarrow \Omega)$ and it is called simple if it is one-to-one. Assume $\sum(\psi, \Omega)=\left\{\psi \in \operatorname{Lip}(\overline{\mathcal{B}} \rightarrow \Omega): \phi_{\mid \partial \mathcal{B}}=\psi\right\}$. For any simple, closed and rectifiable curve in $\Omega, \sigma(\psi, \Omega)$ is a nonvoid set provided that $\Omega$ is an open domain which is simply connected.

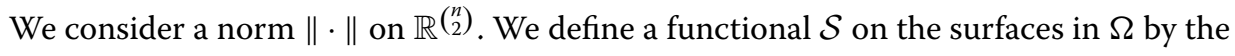
following relation:

$$
\mathcal{S} \phi=\int_{\mathcal{B}}\left\|P \cdot\left(\frac{\partial \phi}{\partial u_{1}} \wedge \frac{\partial \phi}{\partial u_{2}}\right)\right\| d u
$$

In which the mapping $u \rightarrow \phi(u)$ is Lipschitzian on $\overline{\mathcal{B}}$, and $\frac{\partial \phi}{\partial u_{1}} \wedge \frac{\partial \phi}{\partial u_{2}}$ is the wedge product

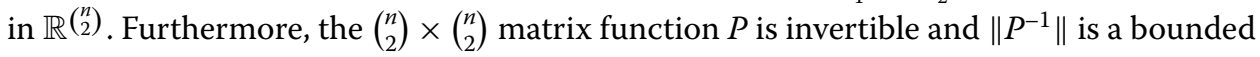
function on $\phi(\overline{\mathcal{B}})$. The following result is stated in [6].

Consider a $C^{1}$ function on the set $\Omega \subseteq \mathbb{R}^{n}$ such as $x \rightarrow f(x) \in \mathbb{R}^{n}$ and the following ODE system:

$$
\frac{d x}{d t}=f(x)
$$


We consider the function $\phi_{t}(u)=x(t, \phi(u))$ as the solution of (5.2), passing through $(0 ; \phi(u))$, for any $\phi$. We define the right-hand derivative of $\mathcal{S} \phi_{t}$, by the following relation:

$$
D_{+} \mathcal{S} \phi_{t}=\int_{\overline{\mathcal{B}}} \lim _{h \rightarrow 0^{+}} \frac{1}{h}\left(\left\|z+h Q\left(\phi_{t}(u)\right) z\right\|-\|z\|\right) d u .
$$

In which $Q=P_{f} P^{-1}+P \frac{\partial f[2]}{\partial x} P^{-1}, P_{f}$ represents the directional derivative of $P$ in the direction of the vector field $f$, and $\frac{\partial f}{\partial x}$ denotes the second additive compound matrix of $\frac{\partial f}{\partial x}$. Furthermore, we consider the following differential equation:

$$
\frac{d z}{d t}=Q\left(\phi_{t}(u)\right) z
$$

For which the solution is of the form $z=P .\left(\frac{\partial \phi}{\partial u_{1}} \wedge \frac{\partial \phi}{\partial u_{2}}\right)$. The formula $D_{+} \mathcal{S} \phi_{t}$ can be expressed as,

$$
D_{+} \mathcal{S} \phi_{t}=\int_{\overline{\mathcal{B}}} D_{+}\|z\| d u
$$

Let $P$ be the following matrix:

$$
P=\left[\begin{array}{ccc}
e^{3 c+3 g} & 0 & 0 \\
0 & e^{3 c+3 g} & 0 \\
0 & 0 & e^{3 c+3 g}
\end{array}\right]
$$

Therefore, we have the matrix

$$
P_{f} P^{-1}=\operatorname{diag}\left(3 c^{\prime}+3 g^{\prime}, 3 c^{\prime}+3 g^{\prime}, 3 c^{\prime}+3 g^{\prime}\right)
$$

hence the matrix $P J^{[2]} P^{-1}=J^{[2]}$ and

$$
Q=P_{f} P^{-1}+P J^{[2]} P^{-1}=\left[\begin{array}{lll}
A_{11} & A_{12} & A_{13} \\
A_{21} & A_{22} & A_{23} \\
A_{31} & A_{32} & A_{33}
\end{array}\right],
$$

where

$$
\begin{aligned}
A_{11}= & \beta_{1}-2 \beta_{1} c^{2}-\beta_{1} g-\beta_{1} f+\delta g+\beta_{2}-\beta_{2} c \\
& -2 \beta_{2} g^{2}-\beta_{2} f-\delta c-2 \mu-2+\beta_{1} c-2 \beta_{1} g c \\
& -2 \beta_{1} c f-3(\mu+1) c+\beta_{2} g-2 \beta_{2} g c-2 \beta_{2} g f-3(\mu+1) g, \\
A_{12}= & \beta_{2}\left(c g+g^{2}-g+g f\right)+\delta c g, \\
A_{13}= & \beta_{1}\left(c-c^{2}-c f-c g\right)+\delta c g, \\
A_{21}= & \alpha_{2}, \\
A_{22}= & \beta_{1}+\beta_{1} c-2 \beta_{1} c^{2}-\beta_{1} g-2 \beta_{1} c g-\beta_{1} f-2 \beta_{1} c f \\
& +\delta g-2 \mu-1-\delta c g-3(\mu+1) c-3 \beta_{2} t^{2}-3 \beta_{2} c g \\
& -3 \beta_{2} f g-3(\mu+1) g+3 \beta_{2} g,
\end{aligned}
$$




$$
\begin{aligned}
A_{23}= & -\beta_{1} c+\beta_{1} c g+\beta_{1} c^{2}+\beta_{1} c f+\delta c-\delta g c, \\
A_{31}= & -\alpha_{1}, \\
A_{32}= & -\beta_{2} g+\beta_{2} g^{2}+\beta_{2} c g+\beta_{2} g f-\delta g+\delta g c, \\
A_{33}= & \beta_{2}-\beta_{2} c-2 \beta_{2} c g-2 \beta_{2} g-2 \beta_{2} g^{2}-\beta_{2} f-2 \beta_{2} g f \\
& -\delta c+\delta c g-2 \mu-1+3 \beta_{1} c-3 \beta_{1} c^{2}-3 \beta_{1} g c-3 \beta_{1} c f \\
& -3(\mu+1) c+3 \beta_{2} g-3(\mu+1) g .
\end{aligned}
$$

We use the following norm for $z=\left(z_{1}, z_{2}, z_{3}\right)^{T}$ :

$$
\|z\|= \begin{cases}\max \left\{\left|z_{1}\right|+\left|z_{3}\right|,\left|z_{2}\right|+\left|z_{3}\right|\right\} & \text { if } z_{2} z_{3} \geq 0 \\ \max \left\{\left|z_{1}\right|+\left|z_{3}\right|,\left|z_{2}\right|\right\} & \text { if } z_{2} z_{3} \leq 0\end{cases}
$$

Lemma 5.1 There exists a constant $\tau>0$ such that $D_{+}\|z\| \leq-\tau\|z\|$ for all $z \in \mathbb{R}^{3}$ and all $c, g, f \geq 0$, where $z$ is a solution of $\frac{d z}{d t}=Q\left(\phi_{t}(u)\right) z$ provided

$$
\left\{\begin{array}{l}
2 \delta<\beta_{1} \\
\beta_{2}+\alpha_{1}<1 \\
\delta+3 \beta_{2}<3
\end{array}\right.
$$

Proof We prove the existence of some $\tau>0$ such that $D_{+}\|z\| \leq-\tau\|z\|$ and $z$ is a solution of $\frac{d z}{d t}=Q\left(\phi_{t}(u)\right) z$. The proof contains eight cases based on the different octants and the definition of the norm in (5.6). We consider $\delta=\delta_{1} \beta_{1}-\delta_{2} \beta_{2}>0$. By using Theorem 3.6, we have $\beta_{1}<\beta_{2}$ and $\alpha_{2}<\alpha_{1}$. For $\delta<0$, the proof is similar.

Case 1: $z_{1}, z_{2}, z_{3}>0$ and $\left|z_{1}\right|+\left|z_{3}\right|>\left|z_{2}\right|+\left|z_{3}\right|$. In this case $\|z\|=\left|z_{1}\right|+\left|z_{3}\right|$

$$
\begin{aligned}
D_{+}\|z\|= & D_{+}\left(\left|z_{1}\right|+\left|z_{3}\right|\right) \\
= & D_{+}\left(z_{1}+z_{3}\right) \\
= & z_{1}^{\prime}+z_{3}^{\prime} \\
= & \left(A_{11}+A_{31}\right) z_{1}+\left(A_{12}+A_{32}\right) z_{2}+\left(A_{13}+A_{33}\right) z_{3} \\
= & {\left[\beta_{1}-2 \beta_{1} c^{2}-\beta_{1} g-\beta_{1} f+\delta g+\beta_{2}-\beta_{2} c-2 \beta_{2} g^{2}\right.} \\
& -\beta_{2} f-\delta c-2 \mu-2+\beta_{1} c-2 \beta_{1} g c-2 \beta_{1} c f+\beta_{2} g \\
& \left.-3(\mu+1) c-2 \beta_{2} g c-2 \beta_{2} g f-3(\mu+1) g-\alpha_{1}\right] z_{1} \\
& +\left[2 \beta_{2} c g+2 \beta_{2} g^{2}-2 \beta_{2} g+2 \beta_{2} g f+2 \delta c g-\delta g\right] z_{2} \\
& +\left[\beta_{1} c-\beta_{1} c^{2}-\beta_{1} c f-\beta_{1} c g+2 \delta c g+\beta_{2}-\beta_{2} c-2 \beta_{2} c g\right. \\
& -2 \beta_{2} g-2 \beta_{2} g^{2}-\beta_{2} f-2 \beta_{2} g f-\delta c-2 \mu-1+3 \beta_{2} g \\
& \left.+3 \beta_{1} c-3 \beta_{1} c^{2}-3 \beta_{1} g c-3 \beta_{1} c f-3(\mu+1) c-3(\mu+1) g\right] z_{3} .
\end{aligned}
$$


We consider $K_{1}=2 \beta_{2} c g+2 \beta_{2} g^{2}+2 \beta_{2} g f+2 \delta c g$, since $\left|z_{2}\right|<\left|z_{1}\right|$, therefore, $K_{1}\left|z_{2}\right|<K_{1}\left|z_{1}\right|$. We have

$$
\begin{aligned}
D_{+}\|z\| \leq & {\left[\left(\beta_{1}+\beta_{2}-2\right)-\alpha_{1}+\left(\delta-\beta_{1}\right) g-2 \beta_{1} c^{2}-\beta_{1} f-\beta_{2} c\right.} \\
& +\left(\beta_{1}-3\right) c-\beta_{2} f-\delta c-2 \mu+2\left(\delta-\beta_{1}\right) g c-2 \beta_{1} c f-3 \mu c \\
& \left.+\left(\beta_{2}-3\right) g-3 \mu g\right]\left|z_{1}\right| \\
& +\left[\left(\beta_{1}-\beta_{2}\right) c+\left(3 \beta_{1}-3\right) c+2\left(\delta-\beta_{2}\right) c g+\left(\beta_{2}-3\right) g-3 \mu g\right. \\
& -4 \beta_{1} c^{2}-4 \beta_{1} c f+\left(\beta_{2}-1\right)-2 \mu-4 \beta_{1} c g-2 \beta_{1} g^{2}-\beta_{2} f \\
& \left.-2 \beta_{2} g f-\delta c-3 \mu c\right]\left|z_{3}\right| \\
\leq & \max \left\{L_{11}, L_{12}\right\}\|z\|,
\end{aligned}
$$

where

$$
\begin{aligned}
L_{11}= & \left(\beta_{1}+\beta_{2}-2\right)-\alpha_{1}+\left(\delta-\beta_{1}\right) g-2 \beta_{1} c^{2}-\beta_{1} f-\beta_{2} c \\
& +\left(\beta_{1}-3\right) c-\beta_{2} f-\delta c-2 \mu+2\left(\delta-\beta_{1}\right) g c-2 \beta_{1} c f-3 \mu c \\
& +\left(\beta_{2}-3\right) g-3 \mu g, \\
L_{12}= & \left(\beta_{1}-\beta_{2}\right) c+\left(3 \beta_{1}-3\right) c+2\left(\delta-\beta_{2}\right) c g+\left(\beta_{2}-3\right) g-3 \mu g \\
& -4 \beta_{1} c^{2}-4 \beta_{1} c f+\left(\beta_{2}-1\right)-2 \mu-4 \beta_{1} c g-2 \beta_{1} g^{2}-\beta_{2} f \\
& -2 \beta_{2} g f-\delta c-3 \mu c .
\end{aligned}
$$

$D_{+}\|z\|$ be bounded away from zero on the negative side for all $z$ and $c, g, f>0$, therefore, we require

$$
\left\{\begin{array}{l}
\beta_{1}+\beta_{2}<2 \\
\beta_{2}<1 \\
\delta<\beta_{1} .
\end{array}\right.
$$

Case 2: $z_{1}, z_{2}, z_{3}>0$ and $\left|z_{1}\right|+\left|z_{3}\right|<\left|z_{2}\right|+\left|z_{3}\right|$. In this case $\|z\|=\left|z_{2}\right|+\left|z_{3}\right|$ and

$$
\begin{aligned}
D_{+}\|z\|= & D_{+}\left(\left|z_{2}\right|+\left|z_{3}\right|\right) \\
= & D_{+}\left(z_{2}+z_{3}\right) \\
= & z_{2}^{\prime}+z_{3}^{\prime} \\
= & \left(A_{21}+A_{31}\right) z_{1}+\left(A_{22}+A_{32}\right) z_{2}+\left(A_{23}+A_{33}\right) z_{3} \\
= & {\left[\alpha_{2}-\alpha_{1}\right] z_{1}+\left[\left(\beta_{1}+\beta_{1} c-2 \beta_{1} c^{2}-\beta_{1} g-2 \beta_{1} g c\right.\right.} \\
& -\beta_{1} f-2 \beta_{1} c f-2 \mu-1-3(\mu+1) c-2 \beta_{2} g^{2}+2 \beta_{2} g \\
& \left.-2 \beta_{2} g c-2 \beta_{2} g f-3(\mu+1) g\right] z_{2}+\left[2 \beta_{1} c+\beta_{2}-\beta_{2} c\right. \\
& -2 \beta_{2} c g-2 \beta_{2} g^{2}-\beta_{2} f-2 \beta_{2} g f-2 \mu-1-2 \beta_{1} c f \\
& \left.-3(\mu+1) c-3(\mu+1) g-2 \beta_{1} g c-2 \beta_{1} c^{2}+\beta_{2} g\right] z_{3} .
\end{aligned}
$$


Since the coefficient $z_{1}$ is smaller than $\alpha_{2}$ and $z_{1}<z_{2}$,

$$
\begin{aligned}
D_{+}\|z\| \leq & {\left[\left(\alpha_{2}+\beta_{1}-1\right)-2 \mu+\left(\beta_{1}-3\right) c-3 \mu c+\left(2 \beta_{2}-3\right) g-3 \mu g\right.} \\
& \left.-2 \beta_{1} g c-2 \beta_{2} g c-2 \beta_{2} g^{2}-2 \beta_{2} g f-2 \beta_{1} c^{2}-\beta_{1} g-\beta_{1} f-2 \beta_{1} c f\right] z_{2} \\
& +\left[\left(\beta_{2}-1\right)-2 \mu+\left(\beta_{2}-3\right) g-3 \mu g+\left(2 \beta_{1}-3\right) c-\beta_{2} c-2 \beta_{2} c g\right. \\
& \left.-2 \beta_{2} g^{2}-\beta_{2} f-2 \beta_{2} g f-2 \beta_{1} g c-2 \beta_{1} c f-3 \mu c-2 \beta_{1} c^{2}\right] z_{3} \\
\leq & \max \left\{L_{21}, L_{22}\right\}\|z\| .
\end{aligned}
$$

Here

$$
\begin{aligned}
L_{21}= & \left(\alpha_{2}+\beta_{1}-1\right)-2 \mu+\left(\beta_{1}-3\right) c-3 \mu c+\left(2 \beta_{2}-3\right) g-3 \mu g \\
& -2 \beta_{1} g c-2 \beta_{2} g c-2 \beta_{2} g^{2}-2 \beta_{2} g f-2 \beta_{1} c^{2}-\beta_{1} g-\beta_{1} f-2 \beta_{1} c f, \\
L_{22}= & \left(\beta_{2}-1\right)-2 \mu+\left(\beta_{2}-3\right) g-3 \mu g+\left(2 \beta_{1}-3\right) c-\beta_{2} c-2 \beta_{2} c g \\
& -2 \beta_{2} g^{2}-\beta_{2} f-2 \beta_{2} g f-2 \beta_{1} g c-2 \beta_{1} c f-3 \mu c-2 \beta_{1} c^{2} .
\end{aligned}
$$

$D_{+}\|z\|$ be bounded away from zero on the negative side for all $z$ and $c, g, f>0$, therefore, we require

$$
\left\{\begin{array}{l}
\beta_{1}+\alpha_{2}<1 \\
\beta_{2}<1
\end{array}\right.
$$

Case 3: $z_{1}<0<z_{2}, z_{3}$ and $\left|z_{1}\right|+\left|z_{3}\right|>\left|z_{2}\right|+\left|z_{3}\right|$, therefore $\|z\|=\left|z_{1}\right|+\left|z_{3}\right|$ and

$$
\begin{aligned}
D_{+}\|z\|= & D_{+}\left(\left|z_{1}\right|+\left|z_{3}\right|\right) \\
= & D_{+}\left(-z_{1}+z_{3}\right) \\
= & -z_{1}^{\prime}+z_{3}^{\prime} \\
= & \left(A_{31}-A_{11}\right) z_{1}+\left(A_{32}-A_{12}\right) z_{2}+\left(A_{33}-A_{13}\right) z_{3} \\
= & -\alpha_{1}-\beta_{1}+2 \beta_{1} c^{2}+\beta_{1} g+\beta_{1} f-\delta g-\beta_{2}+\beta_{2} c \\
& +2 \beta_{2} g^{2}+\beta_{2} f+\delta c+2 \mu+2-\beta_{1} c+2 \beta_{1} g c+2 \beta_{1} c f \\
& \left.+3(\mu+1) c-\beta_{2} g+2 \beta_{2} g c+2 \beta_{2} g f+3(\mu+1) g\right] z_{1} \\
& \times(-\delta g) z_{2}+\left[\beta_{2}-\beta_{2} c-2 \beta_{2} c g-2 \beta_{2} g-2 \beta_{2} g^{2}-\beta_{2} f\right. \\
& -2 \beta_{2} g f-\delta c-2 \mu-1+2 \beta_{1} c-2 \beta_{1} c^{2}-2 \beta_{1} g c \\
& \left.-2 \beta_{1} c f-3(\mu+1) c-3(\mu+1) g\right] z_{3} .
\end{aligned}
$$

Since $\left|z_{2}\right|<\left|z_{1}\right|$, we have

$$
\begin{aligned}
D_{+}\|z\| \leq & {\left[\left(\alpha_{1}+\beta_{1}+\beta_{2}-2\right)-2 \mu+\left(\delta-\beta_{1}\right) t+\left(\beta_{1}-3\right) c-3 \mu c\right.} \\
& +\left(\beta_{2}-3\right) g-3 \mu g-2 \beta_{1} c^{2}-\beta_{1} f-\beta_{2} c-2 \beta_{2} g^{2}-\beta_{2} f
\end{aligned}
$$




$$
\begin{aligned}
& \left.-\delta c-2 \beta_{1} g c-2 \beta_{1} c f-2 \beta_{2} g c-2 \beta_{2} g f\right]\left|z_{1}\right| \\
& +\left[\left(\beta_{2}-1\right)-2 \mu+\left(2 \beta_{1}-3\right) c-\beta_{2} c-2 \beta_{1} c f-2 \beta_{1} c^{2}\right. \\
& -2 \beta_{1} c g+\left(\beta_{2}-3\right) g-3 \mu g-2 \beta_{2} g^{2}-\beta_{2} f-2 \beta_{2} c g-\delta c \\
& \left.-2 \beta_{2} g f-3 \mu c\right] z_{3} \leq \max \left\{L_{31}, L_{32}\right\}\|z\| .
\end{aligned}
$$

Here

$$
\begin{aligned}
L_{31}= & \left(\alpha_{1}+\beta_{1}+\beta_{2}-2\right)-2 \mu+\left(\delta-\beta_{1}\right) t+\left(\beta_{1}-3\right) c-3 \mu c \\
& +\left(\beta_{2}-3\right) g-3 \mu g-2 \beta_{1} c^{2}-\beta_{1} f-\beta_{2} c-2 \beta_{2} g^{2}-\beta_{2} f \\
& -\delta c-2 \beta_{1} g c-2 \beta_{1} c f-2 \beta_{2} g c-2 \beta_{2} g f, \\
L_{32}= & \left(\beta_{2}-1\right)-2 \mu+\left(2 \beta_{1}-3\right) c-\beta_{2} c-2 \beta_{1} c f-2 \beta_{1} c^{2} \\
& -2 \beta_{1} c g+\left(\beta_{2}-3\right) g-3 \mu g-2 \beta_{2} g^{2}-\beta_{2} f-2 \beta_{2} c g-\delta c \\
& -2 \beta_{2} g f-3 \mu c .
\end{aligned}
$$

Therefore, we require

$$
\left\{\begin{array}{l}
\alpha_{1}+\beta_{1}+\beta_{2}<2 \\
\beta_{2}<1 \\
\delta<\beta_{1}
\end{array}\right.
$$

Case 4: $z_{1}<0<z_{2}, z_{3}$ and $\left|z_{1}\right|+\left|z_{3}\right|<\left|z_{2}\right|+\left|z_{3}\right|$. Hence $\|z\|=\left|z_{2}\right|+\left|z_{3}\right|$ and $D_{+}\|z\|=$ $D_{+}\left(\left|z_{2}\right|+\left|z_{3}\right|\right)$. We have

$$
\begin{aligned}
D_{+}\|z\|= & D_{+}\left(\left|z_{2}\right|+\left|z_{3}\right|\right) \\
= & D_{+}\left(z_{2}+z_{3}\right) \\
= & z_{2}^{\prime}+z_{3}^{\prime} \\
= & \left(A_{21}+A_{31}\right) z_{1}+\left(A_{22}+A_{32}\right) z_{2}+\left(A_{23}+A_{33}\right) z_{3} \\
= & {\left[\alpha_{2}-\alpha_{1}\right] z_{1}+\left[\beta_{1}+\beta_{1} c-2 \beta_{1} c^{2}-\beta_{1} g-2 \beta_{1} c g-\beta_{1} f\right.} \\
& -2 \beta_{1} c f+\delta g-2 \mu-1-3(\mu+1) c-2 \beta_{2} g^{2}-2 \beta_{2} c g-\delta g \\
& \left.-2 \beta_{2} f g-3(\mu+1) t+2 \beta_{2} g\right] z_{2}+\left[2 \beta_{1} c-2 \beta_{1} c g+\beta_{2}-\beta_{2} c\right. \\
& -2 \beta_{2} c g-2 \beta_{2} g-2 \beta_{2} g^{2}-\beta_{2} f-2 \beta_{2} g f-2 \mu-1-2 \beta_{1} c^{2} \\
& \left.-3(\mu+1) c+3 \beta_{2} g-2 \beta_{1} c f-3(\mu+1) g\right] z_{3} .
\end{aligned}
$$

Since $\left|z_{1}\right|<\left|z_{2}\right|, \alpha_{1}\left|z_{1}\right|<\alpha_{1}\left|z_{2}\right|$, we have

$$
\begin{aligned}
D_{+}\|z\|< & {\left[\left(\alpha_{1}+\beta_{1}-1\right)-2 \mu+\left(\beta_{1}-3\right) c-3 \mu c+\left(2 \beta_{2}-3\right) g-3 \mu g\right.} \\
& \left.-2 \beta_{1} c^{2}-\beta_{1} g-2 \beta_{1} g c-\beta_{1} f-2 \beta_{1} c f-2 \beta_{2} g^{2}-2 \beta_{2} g c-2 \beta_{2} g f\right] z_{2} \\
& +\left[-2 \beta_{1} g c-2 \beta_{2} g c-2 \beta_{1} c^{2}-2 \beta_{1} c f+\left(3 \beta_{2}-3\right) g-3 \mu g-2 \mu\right.
\end{aligned}
$$




$$
\begin{aligned}
& \left.-\beta_{2} f-2 \beta_{2} g^{2}+\left(2 \beta_{1}-3\right) c-\beta_{2} c+\left(\beta_{2}-1\right)-2 \beta_{2} g f-3 \mu c\right] z_{3} \\
\leq & \max \left\{L_{41}, L_{42}\right\}\|z\| .
\end{aligned}
$$

Here

$$
\begin{aligned}
L_{41}= & \left(\alpha_{1}+\beta_{1}-1\right)-2 \mu+\left(\beta_{1}-3\right) c-3 \mu c+\left(2 \beta_{2}-3\right) g-3 \mu g \\
& -2 \beta_{1} c^{2}-\beta_{1} g-2 \beta_{1} g c-\beta_{1} f-2 \beta_{1} c f-2 \beta_{2} g^{2}-2 \beta_{2} g c-2 \beta_{2} g f, \\
L_{42}= & -2 \beta_{1} g c-2 \beta_{2} g c-2 \beta_{1} c^{2}-2 \beta_{1} c f+\left(3 \beta_{2}-3\right) g-3 \mu g-2 \mu \\
& -\beta_{2} f-2 \beta_{2} g^{2}+\left(2 \beta_{1}-3\right) c-\beta_{2} c+\left(\beta_{2}-1\right)-2 \beta_{2} g f-3 \mu c .
\end{aligned}
$$

Therefore, we require

$$
\left\{\begin{array}{l}
\alpha_{1}+\beta_{1}<1 \\
\beta_{2}<\alpha_{2} \\
\beta_{2}<1
\end{array}\right.
$$

Case 5: $z_{2}<0<z_{1}, z_{3}$ and $\left|z_{1}\right|+\left|z_{3}\right|>\left|z_{2}\right|$. Hence $\|z\|=\left|z_{1}\right|+\left|z_{3}\right|$ and

$$
\begin{aligned}
D_{+}\|z\|= & D_{+}\left(\left|z_{1}\right|+\left|z_{3}\right|\right)=D_{+}\left(z_{1}+z_{3}\right)=z_{1}^{\prime}+z_{3}^{\prime} \\
= & \left(A_{11}+A_{31}\right) z_{1}+\left(A_{12}+A_{32}\right) z_{2}+\left(A_{13}+A_{33}\right) z_{3} \\
= & {\left[\beta_{1}-2 \beta_{1} c^{2}-\beta_{1} g-\beta_{1} f+\delta g+\beta_{2}-\beta_{2} c-2 \beta_{2} g^{2}\right.} \\
& -\beta_{2} f-\delta c-2 \mu-2+\beta_{1} c-2 \beta_{1} g c-2 \beta_{1} c f+\beta_{2} g \\
& \left.-3(\mu+1) c-2 \beta_{2} g c-2 \beta_{2} g f-3(\mu+1) g-\alpha_{1}\right] z_{1} \\
& +\left[2 \beta_{2} c g+2 \beta_{2} g^{2}-2 \beta_{2} g+2 \beta_{2} g f+2 \delta c g-\delta g\right] z_{2} \\
& +\left[4 \beta_{1} c-4 \beta_{1} c^{2}-4 \beta_{1} c f-4 \beta_{1} c g+2 \delta c g+\beta_{2}\right. \\
& -\beta_{2} c-2 \beta_{2} c g+\beta_{2} g-2 \beta_{2} g^{2}-\beta_{2} f-2 \beta_{2} g f \\
& -\delta c-2 \mu-1-3(\mu+1) c-3(\mu+1) g] z_{3} .
\end{aligned}
$$

We consider $K_{5}=2 \beta_{2} g+\delta g$. Therefore $K_{5}\left|z_{2}\right|<K_{5}\left|z_{1}\right|+K_{5}\left|z_{3}\right|$. Hence we have

$$
\begin{aligned}
D_{+}\|z\|< & {\left[\left(\beta_{1}+\beta_{2}-2\right)-2 \mu-2 \beta_{1} c^{2}+\left(2 \delta-\beta_{1}\right) g-\beta_{1} f-\beta_{2} c-2 \beta_{2} g^{2}\right.} \\
& -\beta_{2} f-\delta c+\left(\beta_{1}-3\right) c-3 \mu c-2 \beta_{1} g c-2 \beta_{1} c f+\left(3 \beta_{2}-3\right) g-3 \mu g \\
& \left.-2 \beta_{2} g c-2 \beta_{2} g f-\alpha_{1}\right]\left|z_{1}\right|+\left[\left(3 \beta_{1}-3\right) c+\left(\beta_{1}-\beta_{2}\right) c-4 \beta_{1} c^{2}-4 \beta_{1} c f\right. \\
& +2\left(\delta-\beta_{2}\right) c g+\left(\beta_{2}-1\right)-2 \mu-4 \beta_{1} c g+\left(\delta+3 \beta_{2}-3\right) g-3 \mu g-2 \beta_{2} g^{2} \\
& \left.-\beta_{2} f-2 \beta_{2} g f-\delta c-3 \mu c\right] z_{3} \\
\leq & \max \left\{L_{51}, L_{52}\right\}\|z\| .
\end{aligned}
$$


Here

$$
\begin{aligned}
L_{51}= & \left(\beta_{1}+\beta_{2}-2\right)-2 \mu-2 \beta_{1} c^{2}+\left(2 \delta-\beta_{1}\right) g-\beta_{1} f-\beta_{2} c-2 \beta_{2} g^{2} \\
& -\beta_{2} f-\delta c+\left(\beta_{1}-3\right) c-3 \mu c-2 \beta_{1} g c-2 \beta_{1} c f+\left(3 \beta_{2}-3\right) g-3 \mu g \\
& -2 \beta_{2} g c-2 \beta_{2} g f-\alpha_{1}, \\
L_{52}= & \left(3 \beta_{1}-3\right) c+\left(\beta_{1}-\beta_{2}\right) c-4 \beta_{1} c^{2}-4 \beta_{1} c f \\
& +2\left(\delta-\beta_{2}\right) c g+\left(\beta_{2}-1\right)-2 \mu-4 \beta_{1} c g+\left(\delta+3 \beta_{2}-3\right) g-3 \mu g-2 \beta_{2} g^{2} \\
& -\beta_{2} f-2 \beta_{2} g f-\delta c-3 \mu c .
\end{aligned}
$$

Therefore, we require

$$
\left\{\begin{array}{l}
\beta_{2}<1 \\
\beta_{2}+\beta_{1}<2 \\
2 \delta<\beta_{1} \\
\delta+3 \beta_{2}<3
\end{array}\right.
$$

Case 6: $z_{2}<0<z_{1}, z_{3}$ and $\left|z_{1}\right|+\left|z_{3}\right|<\left|z_{2}\right|$. Hence $\|z\|=\left|z_{2}\right|$ and

$$
\begin{aligned}
D_{+}\|z\|= & D_{+}\left(\left|z_{2}\right|\right)=-z_{2}^{\prime} \\
= & -A_{21} z_{1}-A_{22} z_{2}-A_{23} z_{3} \\
= & -\alpha_{2} z_{1}+\left[-\beta_{1}-\beta_{1} c+2 \beta_{1} c^{2}+\beta_{1} g+2 \beta_{1} c g\right. \\
& +\beta_{1} f+2 \beta_{1} c f-\delta g+2 \mu+1-2 \delta c g+3(\mu+1) c \\
& \left.+3 \beta_{2} g^{2}+3 \beta_{2} c g+3 \beta_{2} f g+3 \delta g c+3(\mu+1) g-3 \beta_{2} g\right] z_{2} \\
& +\left[\beta_{1} c-\beta_{1} c g-\beta_{1} c^{2}-\beta_{1} c f-\delta c+\delta g c\right] z_{3} .
\end{aligned}
$$

We consider $K_{6}=\beta_{1} c+\delta g c$. Since $\left|z_{1}\right|<\left|z_{2}\right|$ and $\left|z_{3}\right|<\left|z_{2}\right|$, we have $K_{6}\left|z_{3}\right|<K_{6}\left|z_{2}\right|$, therefore

$$
\begin{aligned}
D_{+}\|z\|< & {\left[\left(\beta_{1}-1\right)-2 \mu+\left(2 \beta_{1}-3\right) c-3 \mu c-2 \beta_{1} c^{2}+\left(\delta-\beta_{1}\right) g\right.} \\
& -2 \beta_{1} g c-\beta_{1} f-2 \beta_{1} c f-3 \beta_{2} g^{2}-3 \beta_{2} g c-3 \beta_{2} g f \\
& \left.+\left(3 \beta_{2}-3\right) g-3 \mu g\right]\left|z_{2}\right| .
\end{aligned}
$$

Therefore we require

$$
\left\{\begin{array}{l}
\beta_{2}<1, \\
\delta<\beta_{1} .
\end{array}\right.
$$

Case 7: $z_{3}<0<z_{1}, z_{2}$ and $\left|z_{1}\right|+\left|z_{3}\right|>\left|z_{2}\right|$. Therefore $\|z\|=\left|z_{1}\right|+\left|z_{3}\right|$

$$
\begin{aligned}
D_{+}\|z\| & =D_{+}\left(\left|z_{1}\right|+\left|z_{3}\right|\right) \\
& =z_{1}^{\prime}-z_{3}^{\prime}
\end{aligned}
$$




$$
\begin{aligned}
= & \left(A_{11}-A_{31}\right) z_{1}+\left(A_{12}-A_{32}\right) z_{2}+\left(A_{13}-A_{33}\right) z_{3} \\
= & {\left[\beta_{1}-2 \beta_{1} c^{2}-\beta_{1} g-\beta_{1} f+\delta g+\beta_{2}-\beta_{2} c-2 \beta_{2} g^{2}\right.} \\
& -\beta_{2} f-\delta c-2 \mu-2+\beta_{1} c-2 \beta_{1} g c-2 \beta_{1} c f-3(\mu+1) c \\
& \left.+\beta_{2} g-2 \beta_{2} g c-2 \beta_{2} g f-3(\mu+1) g+\alpha_{1}\right] z_{1}+\delta g z_{2} \\
& \times\left[-2 \beta_{1} c+2 \beta_{1} c^{2}+2 \beta_{1} c f+2 \beta_{1} c g-\beta_{2}+\beta_{2} c+2 \beta_{2} c g\right. \\
& \left.-\beta_{2} g+2 \beta_{2} g^{2}+\beta_{2} f+2 \beta_{2} g f+\delta c+2 \mu+1+3(\mu+1) c+3(\mu+1) t\right] z_{3} .
\end{aligned}
$$

Since $\left|z_{1}\right|+\left|z_{3}\right|>\left|z_{2}\right|$, the relation $\delta g\left|z_{2}\right|<\delta g\left|z_{1}\right|+\delta g\left|z_{3}\right|$ holds, therefore

$$
\begin{aligned}
D_{+}\|z\|= & {\left[\left(\beta_{1}+\beta_{2}+\alpha_{1}-2\right)-2 \mu+\left(\beta_{1}-3\right) c+\left(2 \delta-\beta_{1}\right) g\right.} \\
& +\left(\beta_{2}-3\right) g-3 \mu g-2 \beta_{1} c^{2}-\beta_{1} f-2 \beta_{2} g^{2}-\beta_{2} f-\delta c \\
& \left.-2 \beta_{1} c f-2 \beta_{1} g c-3 \mu c-\beta_{2} c-2 \beta_{2} g c-2 \beta_{2} g f\right] z_{1} \\
& \times\left[\left(\beta_{2}-1\right)-2 \mu+\left(2 \beta_{1}-3\right) c-3 \mu c-\beta_{2} c-2 \beta_{1} c^{2}-2 \beta_{1} c f\right. \\
& -2 \beta_{1} c g-2 \beta_{2} c g+\left(\beta_{2}+\delta-3\right) g-3 \mu g-2 \beta_{2} g^{2}-\beta_{2} f \\
& \left.-2 \beta_{2} g f-\delta c\right]\left|z_{3}\right| \leq \max \left\{L_{71}, L_{72}\right\}\|z\| .
\end{aligned}
$$

Here

$$
\begin{aligned}
L_{71}= & \left(\beta_{1}+\beta_{2}+\alpha_{1}-2\right)-2 \mu+\left(\beta_{1}-3\right) c+\left(2 \delta-\beta_{1}\right) g \\
& +\left(\beta_{2}-3\right) g-3 \mu g-2 \beta_{1} c^{2}-\beta_{1} f-2 \beta_{2} g^{2}-\beta_{2} f-\delta c \\
& -2 \beta_{1} c f-2 \beta_{1} g c-3 \mu c-\beta_{2} c-2 \beta_{2} g c-2 \beta_{2} g f, \\
L_{72}= & \left(\beta_{2}-1\right)-2 \mu+\left(2 \beta_{1}-3\right) c-3 \mu c-\beta_{2} c-2 \beta_{1} c^{2}-2 \beta_{1} c f \\
& -2 \beta_{1} c g-2 \beta_{2} c g+\left(\beta_{2}+\delta-3\right) g-3 \mu g-2 \beta_{2} g^{2}-\beta_{2} f-2 \beta_{2} g f-\delta c .
\end{aligned}
$$

Therefore, we require

$$
\left\{\begin{array}{l}
\beta_{1}+\beta_{2}+\alpha_{1}<2, \\
\beta_{2}<1 \\
2 \delta<\beta_{1} \\
\beta_{2}+\delta<3
\end{array}\right.
$$

Case 8: $z_{3}<0<z_{1}, z_{2}$ and $\left|z_{1}\right|+\left|z_{3}\right|<\left|z_{2}\right|$. Therefore $\|z\|=\left|z_{2}\right|$ and

$$
\begin{aligned}
D_{+}\|z\|= & D_{+}\left(\left|z_{2}\right|\right)=z_{2}^{\prime} \\
= & A_{21} z_{1}+A_{22} z_{2}+A_{23} z_{3} \\
= & \alpha_{2} z_{1}+\left[\beta_{1}+\beta_{1} c-2 \beta_{1} c^{2}-\beta_{1} g-2 \beta_{1} c g-\beta_{1} f\right. \\
& -2 \beta_{1} c f+\delta g-2 \mu-1-\delta c g-3(\mu+1) c-3 \beta_{2} t^{2}
\end{aligned}
$$




$$
\begin{aligned}
& \left.-3 \beta_{2} c g-3 \beta_{2} f g-3(\mu+1) t+3 \beta_{2} t\right] z_{2}\left[-\beta_{1} c+\delta c\right. \\
& \left.+\beta_{1} c g+\beta_{1} c^{2}+\beta_{1} c f-\delta g c\right] z_{3} .
\end{aligned}
$$

We define $K_{8}=\beta_{1} c+\delta g c$. Since $z_{3}<0, \delta>0$, we have $\alpha_{2}\left|z_{1}\right|<\alpha_{2}\left|z_{2}\right|$ and $K_{8}\left|z_{3}\right|<K_{8}\left|z_{2}\right|$ and

$$
\begin{aligned}
D_{+}\|z\| \leq & \left(\left(\alpha_{2}+\beta_{1}-1\right)-2 \mu+\left(2 \beta_{1}-3\right) c-3 \mu c-2 \beta_{1} c^{2}\right. \\
& +\left(3 \beta_{2}-3\right) g-3 \mu g+\left(\delta-\beta_{1}\right) g-2 \beta_{1} g c-\beta_{1} f \\
& \left.-2 \beta_{1} c f-3 \beta_{2} g^{2}-3 \beta_{2} g c-3 \beta_{2} g f\right]\left|z_{2}\right| .
\end{aligned}
$$

Therefore, we require

$$
\left\{\begin{array}{l}
\beta_{1}+\alpha_{2}<1 \\
\delta<\beta_{1} \\
\beta_{2}<1
\end{array}\right.
$$

In [6], the global stability of a unique steady state is investigated by the geometric method. In such cases, a compact absorbing set exists. therefore surfaces remain in $\Omega$ for all times. But there is no such set, when a model such as model (2.5) has backward bifurcation. Hence, the following lemma proves the existence of the sequence $\varphi^{k}$ of surfaces (see [1]).

Lemma 5.2 Suppose that $\psi$ be a simple and closed curve in $\Omega$. There exist $\epsilon>0$ and a surface $\varphi^{k}$ that minimizes $S$ with respect to $\Sigma(\psi, \Omega)$ such that $\varphi_{t}^{k} \subseteq \Omega$ for all $k=1,2,3, \ldots$ and for all $t \in[0, \epsilon]$.

Proof Suppose $\xi=\frac{1}{2} \min \{c:(c, g, f) \in \psi\}$. We consider $\delta>0$, if $\delta<0$ then the proof is similar. In this case

$$
\frac{d c}{d t} \geq-(\mu+1) c
$$

therefore, there exists $\epsilon>0$ such that if $c(0) \geq \xi$ then the solution remains in $\Omega$ for $t \epsilon$ $[0, \epsilon]$. Hence we must show that there exists a sequence $\left\{\varphi^{k}\right\}$ which minimizes $S$ relative to $\Sigma(\psi, \bar{\Omega})$, where $\bar{\Omega}=\{(c, g, f) \in \Omega: c \geq \xi\}$. Let $\varphi(u)=(c(u), g(u), f(u)) \in \Sigma(\psi, \Omega)$, we define a new surface $\tilde{\varphi}(u)=(\tilde{c}(u), \tilde{g}(u), \tilde{f}(u))$, by the following relation:

$$
\tilde{\varphi}(u)= \begin{cases}\varphi(u) & c(u) \geq \xi, \\ (\xi, g, f) & c(u)<\xi \text { and } \xi+g(u)+f(u) \leq 1, \\ \left(\xi, \frac{g}{g+f}(1-\xi), \frac{f}{g+f}(1-\xi)\right) & c(u)<\xi \text { and } \xi+g(u)+f(u)>1,\end{cases}
$$

$\tilde{\varphi}(u) \in \Sigma(\psi, \bar{\Omega})$. Now, we demonstrate that $\mathcal{S} \tilde{\varphi} \leq \mathcal{S} \varphi$ :

$$
\frac{\partial \varphi}{\partial u_{1}} \wedge \frac{\partial \varphi}{\partial u_{2}}=\left[\begin{array}{l}
\frac{\partial c}{\partial u_{1}} \\
\frac{\partial g}{\partial u_{1}} \\
\frac{\partial f}{\partial u_{1}}
\end{array}\right] \wedge\left[\begin{array}{l}
\frac{\partial c}{\partial u_{2}} \\
\frac{\partial g}{\partial u_{2}} \\
\frac{\partial f}{\partial u_{2}}
\end{array}\right]=\left[\begin{array}{l}
\operatorname{det}\left[\begin{array}{ll}
\frac{\partial c}{\partial u_{1}} & \frac{\partial c}{\partial u_{2}} \\
\frac{\partial g}{\partial u_{1}} & \frac{\partial g}{\partial u_{2}} \\
\frac{\partial c}{\partial u_{1}} & \frac{\partial c}{\partial u_{2}} \\
\frac{\partial f}{\partial u_{1}} & \frac{\partial f}{\partial u_{2}} \\
=\frac{\partial g}{\partial u_{1}} & \frac{\partial g}{\partial u_{2}} \\
\frac{\partial f}{\partial u_{1}} & \frac{\partial f}{\partial u_{2}}
\end{array}\right]
\end{array}\right]
$$


is a vector in $\mathbb{R}^{3}$. We denote $\frac{\partial \tilde{\varphi}}{\partial u_{1}} \wedge \frac{\partial \tilde{\varphi}}{\partial u_{2}}=\left(\tilde{x}_{1}, \tilde{x}_{2}, \tilde{x}_{3}\right)^{T}$ and $\frac{\partial \varphi}{\partial u_{1}} \wedge \frac{\partial \varphi}{\partial u_{2}}=\left(x_{1}, x_{2}, x_{3}\right)^{T}$ and show $\left\|\frac{\partial \tilde{\varphi}}{\partial u_{1}} \wedge \frac{\partial \tilde{\varphi}}{\partial u_{1}}\right\| \leq\left\|\frac{\partial \varphi}{\partial u_{1}} \wedge \frac{\partial \varphi}{\partial u_{2}}\right\|$.

Case 1. If $c \geq \xi$ then $\tilde{\varphi}=\varphi$ and $\left|\tilde{x}_{i}\right|=\left|x_{i}\right|(i=1,2,3)$, therefore $\left\|\frac{\partial \tilde{\varphi}}{\partial u_{1}} \wedge \frac{\partial \tilde{\varphi}}{\partial u_{1}}\right\|=\left\|\frac{\partial \varphi}{\partial u_{1}} \wedge \frac{\partial \varphi}{\partial u_{2}}\right\|$. Case 2. If $c<\xi$ and $\xi+g(u)+f(u) \leq 1$, then $\tilde{\varphi}(v)=(\xi, g(v), f(v))$. Therefore

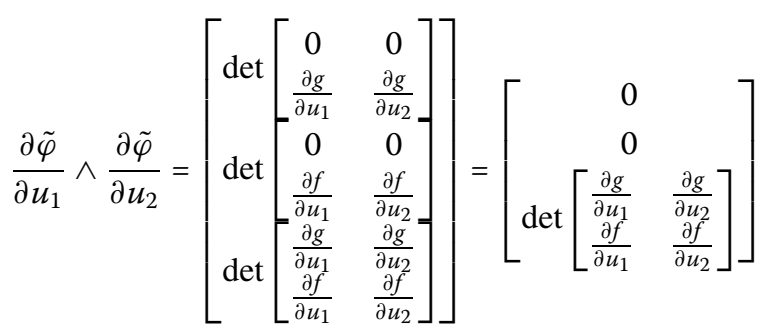

almost everywhere. Hence it follows that $\left|\tilde{x}_{i}\right| \leq\left|x_{i}\right|$, thus $\left\|\frac{\partial \tilde{\varphi}}{\partial u_{1}} \wedge \frac{\partial \tilde{\varphi}}{\partial u_{1}}\right\| \leq\left\|\frac{\partial \varphi}{\partial u_{1}} \wedge \frac{\partial \varphi}{\partial u_{2}}\right\|$.

Case 3. If $c<\xi$ and $\xi+g(u)+f(u)>1$, then $\tilde{\varphi}(v)=\left(\xi, \frac{g}{g+f}(1-\xi), \frac{f}{g+f}(1-\xi)\right)$. Therefore

$$
\frac{\partial \tilde{\varphi}}{\partial u_{j}}=(1-\xi) \frac{f \frac{\partial g}{\partial u_{j}}-g \frac{\partial f}{\partial u_{j}}}{(g+f)^{2}}\left[\begin{array}{c}
0 \\
1 \\
-1
\end{array}\right], \quad j=1,2 .
$$

Thus, $\frac{\partial \tilde{\varphi}}{\partial u_{1}}$ and $\frac{\partial \tilde{\varphi}}{\partial u_{2}}$ are linearly dependent, and hence their wedge product is $\frac{\partial \tilde{\varphi}}{\partial u_{1}} \wedge \frac{\partial \tilde{\varphi}}{\partial u_{2}}=0$. Therefore $\left\|\frac{\partial \tilde{\varphi}}{\partial u_{1}} \wedge \frac{\partial \tilde{\varphi}}{\partial u_{1}}\right\|=0 \leq\left\|\frac{\partial \varphi}{\partial u_{1}} \wedge \frac{\partial \varphi}{\partial u_{2}}\right\|$. We denote $\tilde{i}_{2}(u)=\max \{c, \xi\}$ and hence $\frac{1}{\tilde{c}}<\frac{1}{c}$. Therefore

$$
\begin{aligned}
S \tilde{\varphi} & =\int_{\overline{\mathcal{B}}} \frac{1}{\tilde{i}_{2}}\left\|\frac{\partial \tilde{\varphi}}{\partial u_{1}} \wedge \frac{\partial \tilde{\varphi}}{\partial u_{2}}\right\| d u \\
& \leq \int_{\overline{\mathcal{B}}} \frac{1}{i_{2}}\left\|\frac{\partial \varphi}{\partial u_{1}} \wedge \frac{\partial \varphi}{\partial u_{2}}\right\| d u \\
& =\mathcal{S} \varphi .
\end{aligned}
$$

Suppose that $\left\{\varphi^{k}\right\}$ is a sequence of surfaces that minimizes $\mathcal{S}$ respect to $\Sigma(\psi, \Omega)$. Let $\left\{\tilde{\varphi}^{k}\right\}$ be a sequence of surfaces in $\Sigma(\psi, \tilde{\Omega})$ defined by the above definition. Since $\mathcal{S} \tilde{\varphi} \leq \mathcal{S} \varphi$ for every $k$, and $\Sigma(\psi, \tilde{\Omega})$ is a subset of $\Sigma(\psi, \Omega)$, one concludes that $\tilde{\varphi}^{k}$ minimizes $S$ relative to $\Sigma(\psi, \tilde{\Omega})$.

Lemmas 6.1 and 6.2 and Corollary 5.4 in [1] lead one to conclude to the following theorem.

Theorem 5.3 Positive semi-trajectories of system converges to an equilibrium point, i.e., any $\omega$-limit point of 2.1 in $\Omega$ is an equilibrium point.

Finally, the above theorem implies the following result.

Theorem 5.4 Suppose the inequalities in Lemma 6.2 hold, if $R_{0}>1$, then all solutions of (2.5) tends to the unique positive equilibrium point. 


\section{Example}

In the present study, 30 EFL students at Semnan University participated. They chose modern and traditional education equally. Hence $\beta_{1}=\beta_{2}$. There were 15 students in each group. Before the treatment, all the participants took a reading comprehension test as pre-test. Table 1 shows the results of pre-test.

An independent samples $t$-test was run at the 0.05 level of significance to determine whether the difference between the means was significant or not. The results of the $t$-test presented in Table 2 show that there was no significant difference between the groups as far as their reading comprehension proficiency was concerned before the treatment.

After the treatment, another reading comprehension test was given to the participants. This test served as a post-test. The results of this test are shown in the following tables. As the results in Table 3 show, the modern education group outperformed the traditional education group in post-test. To see if the difference in means was significant, another independent samples $t$-test was run. Table 4 demonstrates the results of the $t$-test for the post-test.

The analysis of the data in Table 4 shows that at the 0.05 level of significance there was a significant difference between the post-test reading mean scores of the subjects from the modern education group and the post-test reading mean scores of the subjects from the traditional education group ( $\mathrm{t}$ observed (3.717) is bigger than $t$ critical (0.001)).

\section{Conclusion}

In this paper, English learning in a university is modeled. This modeling becomes the development of an epidemic model with two strains and superinfection. In most superinfec-

Table 1 Descriptive Statistics of Pre-test in Terms of Groups

\begin{tabular}{lllll}
\hline Variable pretest & Number of subjects & Mean & SD & Std. error mean \\
\hline Modern Education Group & 15 & 14.13 & 2.066 & 0.533 \\
Traditional Education Group & 15 & 14.27 & 2.086 & 0.539 \\
Mean Difference $=-0.14$ & & & & \\
\hline
\end{tabular}

Table 2 Independent samples t-test for pre-test

\begin{tabular}{|c|c|c|c|c|c|c|c|c|c|}
\hline & \multicolumn{2}{|c|}{$\begin{array}{l}\text { Levene's } \\
\text { test for } \\
\text { equality of } \\
\text { variances }\end{array}$} & \multicolumn{7}{|c|}{ Test for equality of means } \\
\hline & \multirow[t]{2}{*}{$\bar{F}$} & \multirow[t]{2}{*}{ sig. } & \multirow[t]{2}{*}{$\bar{t}$} & \multirow[t]{2}{*}{$\mathrm{df}$} & \multirow[t]{2}{*}{ sig(2-tailed) } & \multirow[t]{2}{*}{$\begin{array}{l}\text { Mean } \\
\text { difference }\end{array}$} & \multirow[t]{2}{*}{$\begin{array}{l}\text { SE of } \\
\text { difference }\end{array}$} & \multicolumn{2}{|c|}{$\begin{array}{l}95 \text { percent confidence } \\
\text { interval of the difference }\end{array}$} \\
\hline & & & & & & & & Low & Upper \\
\hline $\begin{array}{l}\text { Equal } \\
\text { variances } \\
\text { assumed }\end{array}$ & 0.131 & 0.720 & -0.176 & 28 & 0.862 & -0.133 & 0.758 & -1.686 & 1.419 \\
\hline
\end{tabular}

Table 3 Descriptive statistics of post-test in terms of groups

\begin{tabular}{lllll}
\hline Variable posttest & Number of subjects & Mean & SD & Std. error mean \\
\hline Modern Education Group & 15 & 18.07 & 1.580 & 0.408 \\
Traditional Education Group & 15 & 16 & 1.464 & 0.378 \\
Mean Difference $=2.07$ & & & \\
\hline
\end{tabular}


Table 4 Independent samples $t$-test for post-test

\begin{tabular}{|c|c|c|c|c|c|c|c|c|c|}
\hline & \multicolumn{2}{|c|}{$\begin{array}{l}\text { Levene's } \\
\text { test for } \\
\text { equality of } \\
\text { variances }\end{array}$} & \multicolumn{7}{|c|}{ Test for equality of means } \\
\hline & \multirow[t]{2}{*}{$F$} & \multirow[t]{2}{*}{ sig. } & \multirow[t]{2}{*}{$t$} & \multirow[t]{2}{*}{$d f$} & \multirow[t]{2}{*}{ sig(2-tailed) } & \multirow[t]{2}{*}{$\begin{array}{l}\text { Mean } \\
\text { difference }\end{array}$} & \multirow[t]{2}{*}{$\begin{array}{l}\text { SE of } \\
\text { difference }\end{array}$} & \multicolumn{2}{|c|}{$\begin{array}{l}95 \text { percent confidence } \\
\text { interval of the difference }\end{array}$} \\
\hline & & & & & & & & Low & Upper \\
\hline $\begin{array}{l}\text { Equal } \\
\text { variances } \\
\text { assumed }\end{array}$ & 0.410 & 0.527 & 3.717 & 28 & 001 & 2.067 & 0.556 & 0.928 & 3.206 \\
\hline
\end{tabular}

tion models, individuals infected with strain two can superinfect individuals infected with strain one. We consider the case in which superinfection goes in both directions.

In this model, individuals can participate in two groups of modern and traditional education based on their abilities and interests. We carried out a qualitative study of this model including the computation of basic reproduction and the existence and locally and globally stable of boundary equilibria and coexistence equilibrium point. The educationfree $E_{0}$ is shown; it is local and global stable under suitable conditions.

By using compound matrices, local and global stability of the coexistence equilibrium point was proven. The global stability conditions of this point indicate that initially, individuals chose the traditional education class more than the modern education class. But eventually the number of modern education graduates was higher.

The analysis of the model showed that the occurrence of backward bifurcation and more complexity can occur when we attempt to eliminate the education problems. Therefore, when $R_{0}<1$, education problems may be persistent.

\section{Acknowledgements}

We would like to thank all who participated in this study and generously gave their time toward this research. We are also grateful for both helpful comments by the anonymous reviewers and guidance from the editorial team.

\section{Funding \\ There is no organization that funded our research.}

Availability of data and materials

Please contact the authors for data requests.

\section{Competing interests}

The authors declare that they have no competing interests.

Authors' contributions

All authors read and approved the final manuscript.

\section{Author details}

'Esfarayen University of Technology, Esfarayen, North Khorasan, Iran. ${ }^{2}$ English Language Dept., Ferdowsi University of Mashhad, Mashhad, Iran.

\section{Publisher's Note}

Springer Nature remains neutral with regard to jurisdictional claims in published maps and institutional affiliations.

Received: 21 July 2020 Accepted: 13 December 2020 Published online: 07 January 2021

References

1. Arino, J., McCluskey, C.C., Van den Driessche, P.: Global results for an epidemic model with vaccination that exhibits backward bifurcation. SIAM J. Appl. Math. 64(1), 260-276 (2003)

2. Castillo-Chavez, C., Song, B.: Dynamical models of tuberculosis and their applications. Math. Biosci. Eng. 2, 361-404 (2004) 
3. Ghasemabadi, A.: Stability and bifurcation in a generalized delay prey-predator model. Nonlinear Dyn. 90, 2239-2251 (2017)

4. Ghasemabadi, A., Rahmanidoust, M.H.: Investigating the dynamics of Lotka-Volterra model with disease in the prey and predator species. Int. J. Nonlinear Anal. Appl. (2019). https://doi.org/10.22075/JJNAA.2019.15881.1832

5. Levine, A., Ferenz, O., Reves, T.: EFL academic reading and modern technology: how can we turn our students into independent critical readers? (2000) TESL-EJ, 4 (4), A-1

6. Li, M.Y., Muldowney, J.S.: A geometric approach to global-stability problems. SIAM J. Math. Anal. 27(4), 1070-1083 (1996)

7. Martcheva, M.: An Introduction to Mathematical Epidemiology. Springer, Berlin (2015)

8. Perko, L.: Differential Equations and Dynamical System. Springer, New York (2000)

Submit your manuscript to a SpringerOpen ${ }^{\circ}$ journal and benefit from:

- Convenient online submission

$\checkmark$ Rigorous peer review

- Open access: articles freely available online

- High visibility within the field

- Retaining the copyright to your article

Submit your next manuscript at $\gg$ springeropen.com 